\title{
Suicide attempts in Chinese Han patients with schizophrenia: cognitive, demographic, and clinical variables
}

\author{
Qilong Dai, ${ }^{1,2}$ Dongmei Wang, ${ }^{1,2}$ Jiesi Wang, ${ }^{1,2}$ Huang $\mathbf{X u},{ }^{1,2}$ Elena C. Andriescue, ${ }^{3}$ \\ Hanjing E. Wu, ${ }^{3}$ Meihong Xiu, ${ }^{4}$ Dachun Chen, ${ }^{4}$ Xiangyang Zhang ${ }^{1,2}$ (iD \\ ${ }^{1}$ CAS Key Laboratory of Mental Health, Institute of Psychology, Chinese Academy of Sciences, Beijing, China. ${ }^{2}$ Department of Psychology, \\ University of Chinese Academy of Sciences, Beijing, China. ${ }^{3}$ Department of Psychiatry and Behavioral Sciences, The University of Texas \\ Health Science Center at Houston, Houston, TX, USA. ${ }^{4}$ Beijing HuiLongGuan Hospital, Peking University, Beijing, China.
}

\begin{abstract}
Objective: The purpose of this study was to investigate the lifetime suicide attempt rate, clinical characteristics and cognitive function of Chinese patients with chronic schizophrenia who had attempted suicide.

Methods: We collected data from 908 schizophrenia inpatients about suicide attempts through interviews with the patients and their families, as well as through medical records. All patients were assessed with the Positive and Negative Syndrome Scale, the Rating Scale for Extrapyramidal Side Effects, the Abnormal Involuntary Movement Scale, and the Repeated Battery for the Assessment of Neuropsychological Status.

Results: Of this sample, 97 (10.68\%) had attempted suicide. Patients who had attempted suicide were younger, had longer illness duration, and more severe general psychopathology and depressive symptoms than those who had not. Logistic regression analysis confirmed that suicide attempts were correlated with age, smoking, and depression. No cognitive performance differences were observed between patients who had and had not attempted suicide.

Conclusions: In China, patients with chronic schizophrenia may have a higher prevalence of lifetime suicide attempts than the general population. Some demographic and clinical variables were related to suicide attempts in patients with chronic schizophrenia.
\end{abstract}

Keywords: Schizophrenia; prevalence; discrepancies; suicide; cognition

\section{Introduction}

Compared to the healthy population, schizophrenia (SCZ) patients are more likely to commit suicide, which is their major cause of death. More than $20 \%$ of SCZ patients have attempted suicide, ${ }^{1}$ and the lifetime suicide mortality rate ranges from 4.9 to $13.9 \% .{ }^{2}$ However, the prevalence of suicidal behavior among SCZ patients varies across the world. For example, the incidence of suicide attempts in SCZ patients ranges from approximately $9 \%$ in Eastern countries to $55 \%$ in Western countries. ${ }^{3} \mathrm{~A}$ previous review showed that suicide attempts, a history of depression, substance abuse, agitation or motor restlessness, fear of further mental deterioration, non-compliance with treatment, and recent experience of loss were associated with suicidal behavior. ${ }^{4}$ These results have been confirmed in subsequent studies, which have suggested that younger age, sex, being single, age of onset, illness insight, and sleep disturbances are also risk factors for suicide. ${ }^{5}$

Correspondence: Xiangyang Zhang, Chinese Academy of Sciences, Institute of Psychology, 16 Lincui Road, Chaoyang District, Beijing 100101, China.

E-mail: zhangxy@psych.ac.cn

Submitted Feb 11 2020, accepted Mar 13 2020, Epub May 112020.
However, since the results of studies on suicide risk factors in SCZ remain inconsistent, ${ }^{6}$ further investigation is required.

Diverse cultures have various effects on suicidal behavior, and cross-cultural research is necessary to better understand suicide attempts. ${ }^{7}$ For example, different traditional values, economic conditions, and understanding of mental health have different effects on suicidal behavior. According to the most recent data from World Health Organization mortality database, suicide rates vary by region, culture, and time period. Suicide rates and male-tofemale suicide ratios also differ between countries with different economic conditions. ${ }^{8}$ Therefore, it is necessary to examine the specific demographic and clinical factors related to suicide in SCZ in different cultures and social environments.

Suicide attempts appear to the best factor for predicting completed suicide. However, few studies have focused on suicide attempts in SCZ patients. ${ }^{8,9}$ Moreover, most
How to cite this article: Dai Q, Wang D, Wang J, $\mathrm{Xu} \mathrm{H}$, Andriescue $\mathrm{EC}$, Wu HE, et al. Suicide attempts in Chinese Han patients with schizophrenia: cognitive, demographic, and clinical variables. Braz J Psychiatry. 2021;43:29-34. http://dx.doi.org/10.1590/15164446-2020-0900 
studies on attempted suicide in SCZ have focused on Caucasian patients. Only a small number of studies have examined the prevalence and clinical characteristics of attempted suicide in Chinese SCZ patients. ${ }^{10}$ Our previous report showed that the prevalence of suicide attempt in chronic SCZ patients was 9.2\%. Moreover, in the Chinese Han population, SCZ patients who attempted suicide appeared to be single, younger, smoke more heavily, and have more hospitalizations and more severe depressive symptoms. ${ }^{9}$ Our recent report showed that the suicide attempt rate of drug-naïve Chinese SCZ patients at the first episode was $12 \%$. Compared with SCZ patients who have not attempted suicide, attempters had higher smoking rates, lower negative symptoms, and better attention. ${ }^{8}$ Attempted suicide may be a risk factor for suicide, or it may be the reason for surviving suicidal behavior. ${ }^{11}$ Therefore, a better understanding of suicide attempts is necessary.

SCZ patients also have significant cognitive impairment, which seriously affects their rehabilitation and daily life. However, whether SCZ patients who have attempted suicide have more severe cognitive impairment remains an open question. Recently, Huber et al. ${ }^{12}$ systematically reviewed studies on the relationship between suicidal behavior and different areas of cognitive impairment, such as memory, language and attention, finding that the cognitive deficits associated with suicidal behavior were independent of mental illness, and the results of each cognitive domain were mixed. The results of studies evaluating suicidal behavior and cognitive performance in chronic SCZ patients have been inconsistent. ${ }^{9,13,14}$ Recently, we investigated the relationship between attempted suicide and cognitive performance on the Repeated Battery for the Assessment of Neuropsychological Status (RBANS) in first-episode and drug-naïve SCZ patients and found that those who had attempted suicide had a better attention score. ${ }^{8}$ Therefore, in this study, we also used the RBANS to verify the hypothesis that higher cognitive performance would be detected in chronic SCZ patients with a history of suicide attempts. In the present study, a large sample of chronic SCZ patients $(n=908)$ was recruited to further investigate the prevalence, traits, and cognitive performance of suicide attempters in a Chinese Han population.

\section{Methods}

\section{Subjects}

We recruited 908 chronic inpatients from Beijing HuiLong-Guan Hospital and Rong-Jun Hospital in Baoding, Hebei Province between December 2006 and December 2008. All patients in both hospitals were screened, and the following criteria were required for inclusion in the study: 1) aged from 25 to 75 years; 2) a SCZ diagnosis according to the DSM-IV Structured Clinical Interview by two independent experienced psychiatrists; 3) an illness duration of $\geqslant 5$ years; 4 ) having taken a fixed dose of an oral antipsychotic drug for more than 6 months prior to inclusion; 5) the ability to provide written informed consent and participate in a clinical assessment. The mean patient age was $47.8 \pm 10.2$ years and the mean illness duration of $24.3 \pm 10.1$ years. All participants had chronic SCZ and had received stable doses of antipsychotic drugs in the last 6 months. Of the 908 patients, 204 were treated with typical antipsychotics, 700 with atypical antipsychotics, and 4 with unrecorded antipsychotics. The antipsychotic drugs included clozapine $(n=415)$, risperidone $(n=194)$, chlorpromazine $(n=65)$, sulpiride $(n=48)$, perphenazine $(n=47)$, quetiapine $(n=40)$, haloperidol $(n=31)$, aripiprazole $(n=28)$, pipotiazine palmitate $(n=13)$, olanzapine $(n=10)$, loxapine $(n=11)$, and ziprasidone $(n=2)$. The daily dose of antipsychotics was converted to an equivalent chlorpromazine dose: the mean daily dose of antipsychotics was $459.07 \pm 426.55 \mathrm{mg} /$ day.

The patients' medical records were obtained and physical examinations were performed. Only those without medical comorbidities and alcohol or substance abuse/ dependence were included in the present study.

We also conducted a precursor study with a similar design in the same hospital. ${ }^{15}$ However, these two studies were totally independent, and the two patient populations did not overlap.

\section{Sociodemographic characteristics}

The researchers applied a detailed questionnaire to obtain data on sociodemographic characteristics, medical and psychological status, smoking behavior, and history of suicide attempts. Supplemental information was obtained from the patients' medical records, families, friends, and clinicians.

According to the World Health Organization, a suicide attempt is defined as a deliberate act of self-destruction, with at least some suicidal intentions, that does not cause death. ${ }^{16}$ In the interview, each patient was asked: "Have you even tried to commit suicide in your life?" If yes, further data was collected on the number of attempts, as well as the method and the exact date of each attempt. The history of suicide attempts was verified through the medical records and clinical diagnostic interviews by qualified psychiatrists with the patients and their families.

\section{Clinical assessment}

To ensure consistent and reliable scores, prior to the study four psychiatrists simultaneously participated in training sessions on the use of the Positive and Negative Syndrome Scale (PANSS) for psychopathological symptoms, the Rating Scale for Extrapyramidal Side Effects (RSESE) for extrapyramidal side effects, and the Abnormal Involuntary Movement Scale (AIMS) for tardive dyskinesia. After training, the interclass correlation coefficients between the four raters on these three rating scales ranged from $0.82-0.86$.

The 30-item Positive and Negative Syndrome Scale (PANSS) was initially classified into three subscales: positive symptoms $(P)$, negative symptoms $(N)$, and general psychopathology (G). ${ }^{17}$ Recently, a five-factor model of PANSS ${ }^{18}$ was put published that included a depression factor (G2, G3, and G6) and a cognitive factor (P2, N5, and G11). 


\section{Cognitive assessment}

To measure cognition, two clinical psychologists applied the RBANS, which consists of 12 subtests that result in five age-adjusted index scores, along with a total score. The five indices included: attention, language, visuospatial/constructional, immediate memory, and delay memory. The RBANS was translated into Chinese by our group, showing good clinical validity and test-retest reliability in both SCZ patients and the general population. ${ }^{19}$ In addition, the two clinical psychologists also participated in a training session on standardized use of the RBANS. In repeated assessments, the intra-rater correlation coefficient was 0.92 .

\section{Statistical analysis}

We described the prevalence rate of suicide attempts among SCZ patients as a percentage, using the chisquare $\left(\chi^{2}\right)$ test to compare the prevalence between male and female patients. Analysis of variance and $\chi^{2}$ were then used to compare the differences in demographic and clinical features between suicide attempters and nonattempters. The Bonferroni correction was used to adjust for multiple tests. Multivariate analysis of covariance was used to examine differences in cognitive performance on the total and five index scores of the RBANS, while adjusting for potential confounding variables. Finally, binary logistic regression was used to examine variables that were significantly correlated with suicide attempts after adjusting for confounders. The data were analyzed in SPSS version 18.0, and two tailed p-values $<0.05$ were considered significant. R version 3.6.2 (http://cran.rproject.org) was used for the power analysis.

\section{Ethics statement}

The institutional review board of Beijing Hui-Long-Guan Hospital approved the research protocol. Each participant provided written informed consent.

\section{Results}

Of the total sample, 97 patients $(10.68 \%)$ had attempted suicide at least once. The attempted suicide rates for male and female patients were $11.05 \%$ (82/742) and $9.04 \%$ (15/166), respectively, with no gender difference among SCZ inpatients $\left(\chi^{2}=0.557\right.$, degrees of freedom [df] $=1, p=0.447)$.

Table 1 shows the sociodemographic and clinical features of all the patients. Compared with non-attempters, the attempters were younger and had a longer illness duration (both $p<0.01$; Bonferroni corrected both $p<0.05$ ). Moreover, the attempters had higher general psychopathology ( $p<0.05$ ) and depression factor scores on the PANSS than non-attempters $(p<0.001)$, and the power varied from 0.68 to 0.99 . The differences in general psychopathology ( $p=0.001$; Bonferroni corrected $p<0.05$ ) and depression factor $(p<0.001$ Bonferroni corrected $p<0.01$ ) between attempters and non-attempters remained significant after adjusting for age and illness duration.

RBANS data were available for 70 patients with a history of suicide attempts and for 510 patients without a history of suicide, but were unavailable for 328 patients.

Table 1 Characteristics of suicide attempters vs. non-attempters among patients with chronic schizophrenia

\begin{tabular}{|c|c|c|c|c|c|}
\hline Characteristic & Suicide attempters $(n=97)$ & Suicide non-attempters $(\mathrm{n}=811)$ & $\mathrm{F} / \chi^{2}$ & df & $\mathrm{p}$-value \\
\hline Male/female & $82 / 15$ & $660 / 151$ & 0.577 & 1 & 0.272 \\
\hline Age (years) & $44.63 \pm 10.497$ & $48.25 \pm 10.118$ & 11.009 & 1.906 & 0.001 \\
\hline Education (years) & $8.88 \pm 3.065$ & $9.23 \pm 6.101$ & 0.312 & 1.896 & 0.577 \\
\hline Marital status & & & 2.982 & 2 & 0.225 \\
\hline Single & 65 & 483 & & & \\
\hline Married & 19 & 159 & & & \\
\hline Divorced & 13 & 166 & & & \\
\hline Smoker/nonsmoker & $71 / 26$ & $531 / 278$ & 2.220 & 1 & 0.136 \\
\hline Age at onset (years) & $22.92 \pm 5.133$ & $23.54 \pm 5.683$ & 1.039 & 1.902 & 0.308 \\
\hline Duration of illness (years) & $21.61 \pm 9.715$ & $24.69 \pm 9.924$ & 8.267 & 1.902 & 0.004 \\
\hline Typical/atypical antipsychotics & $21 / 76$ & $183 / 624$ & 0.010 & 1 & 0.920 \\
\hline $\begin{array}{l}\text { Antipsychotic dose (mg/day) } \\
\text { PANSS }\end{array}$ & $436.80 \pm 304.23$ & $461.74 \pm 438.96$ & 0.290 & 1.888 & 0.591 \\
\hline Positive symptoms & $12.52 \pm 5.140$ & $11.80 \pm 5.045$ & 1.173 & 1.906 & 0.188 \\
\hline Negative symptoms & $21.92 \pm 6.890$ & $22.70 \pm 8.513$ & 0.753 & 1.906 & 0.386 \\
\hline General psychopathology & $26.77 \pm 6.858$ & $25.26 \pm 5.628$ & 5.926 & 1.906 & 0.015 \\
\hline Total score & $61.21 \pm 15.113$ & $59.76 \pm 14.802$ & 0.822 & 1.906 & 0.365 \\
\hline Cognitive factor & $7.36 \pm 3.163$ & $8.02 \pm 3.479$ & 3.154 & 1.906 & 0.076 \\
\hline Depression factor & $4.69 \pm 2.162$ & $3.57 \pm 1.291$ & 54.819 & 1.906 & 0.000 \\
\hline RSESE score & $2.11 \pm 2.313$ & $2.00 \pm 2.620$ & 0.144 & 1.886 & 0.705 \\
\hline AIMS score & $4.87 \pm 5.500$ & $4.72 \pm 4.925$ & 0.074 & 1.820 & 0.786 \\
\hline
\end{tabular}

Data presented as $\mathrm{n}$ or mean \pm standard deviation.

AIMS = Abnormal Involuntary Movement Scale; $\mathrm{df}=$ degrees of freedom; PANSS = Positive and Negative Syndrome Scale; RSESE = Rating Scale for Extrapyramidal Side Effects. 
Table 2 Comparisons of Repeated Battery for the Assessment of Neuropsychological Status scores between patients with chronic schizophrenia with or without a history of suicide attempt

\begin{tabular}{|c|c|c|c|c|c|}
\hline Index & Suicide attempters $(n=70)$ & Suicide non-attempters $(n=510)$ & $\mathrm{F}$ & df & $p$-value \\
\hline Immediate memory & $58.97 \pm 16.869$ & $58.75 \pm 16.450$ & 0.011 & 1.578 & 0.917 \\
\hline Visuospatial skills & $76.89 \pm 19.246$ & $77.92 \pm 18.671$ & 0.189 & 1.578 & 0.664 \\
\hline Language & $80.19 \pm 16.288$ & $81.78 \pm 15.278$ & 0.660 & 1.578 & 0.417 \\
\hline Attention & $68.16 \pm 16.754$ & $71.45 \pm 17.692$ & 2.157 & 1.578 & 0.142 \\
\hline Delayed memory & $66.59 \pm 19.441$ & $66.91 \pm 19.437$ & 0.017 & 1.578 & 0.897 \\
\hline Total score & $63.60 \pm 15.157$ & $64.88 \pm 15.157$ & 0.442 & 1.578 & 0.506 \\
\hline
\end{tabular}

Data presented as mean \pm standard deviation.

$\mathrm{df}=$ degrees of freedom.

Most of these patients refused to take the RBANS test, although some were unable to complete it and others were discharged before taking it. There were no significant differences in demographic or clinical variables between patients who did or did not complete the RBANS test (all $p>0.05$ ).

Table 2 shows that there were no significant differences between the five RBANS domains and total scores (all $p>0.05$ ). After adjusting for age, illness duration, and PANSS general psychopathology score, there were still no differences in RBANS cognitive performance scores between the two groups (all $p>0.05$ ).

Stepwise logistic regression analysis showed that, after controlling for confounding factors, the following variables remained significantly associated with suicide attempts: age (Wald $\chi^{2}=6.48, \mathrm{df}=1, \mathrm{p}=0.011$ ), the PANSS depression factor (Wald $\chi^{2}=31.90, \mathrm{df}=1, \mathrm{p}<0.001$ ), and smoking (Wald $\chi^{2}=4.01 \mathrm{df}=1, \mathrm{p}=0.045$ ).

\section{Discussion}

Few reports have shown sociodemographic and clinical characteristics of Chinese SCZ inpatients with a history of suicide attempt. Our previous study was the first to examine factors related to attempted suicide in SCZ inpatients in a Chinese Han population $(n=520)$, finding that $9.2 \%$ of chronic SCZ patients have attempted suicide. ${ }^{9}$ Moreover, compared to patients who have not attempted suicide, those who have were younger, single, and had more severe depressive symptoms and higher nicotine dependence, as well as more hospitalizations. ${ }^{9}$

In this study, which had a new and larger sample $(n=908)$ of SCZ inpatients, we confirmed a similar prevalence of lifetime suicide attempts $(10.68 \%$.) More importantly, the characteristics for suicide attempters in this study were similar to those of our previous study, ${ }^{9}$ including younger age, longer illness duration, more severe depression, and general psychopathology symptoms, as well as higher smoking rates. Taken together, these two studies indicate that the prevalence of lifetime suicide attempts among hospitalized SCZ patients in the Chinese Han population is comparatively stable (approximately 10\%) and that attempters have similar demographic and clinical features.

In China, suicide is one of main causes of death among the general population, and in 2016, the estimated average national suicide prevalence was 9.7/100,000. ${ }^{7}$ A previous epidemiological survey showed that the lifetime prevalence of suicide attempts was $1 \%$ in metropolitan China, including Beijing and Shanghai. ${ }^{20}$ In this study, the lifetime rate of attempted suicide was approximately 10 times greater in chronic SCZ patients than the general population (10.68 vs. $1 \%$ ). In China, suicide rates are generally higher in rural than urban areas, which is significantly different from Western countries. ${ }^{21}$ Meanwhile, the SCZ prevalence rate is higher in urban than rural areas, although the risk of suicide in SCZ patients is greater in rural areas than in urban areas. ${ }^{22}$ Further investigation of Chinese SCZ patients in both rural and urban areas will be necessary.

In this study, the $10.68 \%$ prevalence of lifetime suicide attempts in chronic SCZ inpatients was much lower than rates reported in the Western countries, which range from 23-48.3\%. ${ }^{23,24}$ Previous studies have reported attempted suicide in 9.7 to $14.6 \%$ of Chinese SCZ patients, ${ }^{9,10,25}$ which is similar to our results. The difference in attempted suicide rates between Chinese and Western SCZ populations might be attributed to the following: 1) in China, nearly one-third of chronic SCZ patients have been treated with clozapine. ${ }^{26} \mathrm{~A}$ recent 10 -year systematic review from 2005 to 2014 confirmed the anti-suicidal effects of clozapine. ${ }^{27}$ Therefore, broad use of clozapine in SCZ treatment could reduce the prevalence of suicide in China; 2) many studies have found that genetic factors significantly influence suicidal behavior - up to $50 \% .^{28}$ Suicide-related polygenes, such as nerve growth factor and brain-derived neurotrophic factor, have different gene variants in Chinese and Caucasian populations, ${ }^{29}$ which could also be a factor in attempted suicide rates; 3 ) in Chinese culture, suiciderelated behaviors are regarded as a sign of weakness, incompetence, and shame, which could prevent patients and family members from reporting a history of suicide attempts, thus leading to underestimation ${ }^{25}$; 4) environmental factors, such as economic conditions, the political environment, and access to mental support systems play an important role in suicidal behavior. Such differences could also lead to a discrepancy in suicide attempt rates. Taken together, the use of clozapine, interethnic genetic differences, and sociocultural environmental factors could account for the lower attempted suicide rate in Chinese SCZ patients.

In this study we found that Chinese SCZ inpatients who had attempted suicide were more likely to smoke, which is consistent with our previous studies in chronic and firstepisode SCZ inpatients. ${ }^{8,9}$ Many previous studies have reported a strong association between smoking and suicidal behavior in SCZ. ${ }^{30}$ After adjusting for relevant suicide factors, smokers had a higher suicide incidence, including a dose-dependent relationship, which suggests 
that smoking could increase suicide risk. ${ }^{30}$ However, the mechanisms underlying the relationship between smoking and suicidal behaviors remain unclear and require further investigation.

SCZ is frequently accompanied by depressive symptoms and depression is highly associated with suicidal behavior. ${ }^{25}$ In this study, PANSS scores for general psychopathology and depression factor differed significantly between those who had and had not attempted suicide, and this association was sustained after Bonferroni correction. This result agreed with our earlier reports on chronic and acute SCZ patients. ${ }^{8,9}$ We also found several other related sociodemographic and clinical factors associated with a history of suicide attempts, such as younger age and longer illness duration, which were consistent with our previous report on chronic SCZ patients, ${ }^{9}$ as well as with findings in Western countries. ${ }^{31}$ However, the results for factors associated with suicide attempts in SCZ patients have been inconsistent. A number of studies have reported other suicide risk factors, such as male gender, unmarried status, hospitalization, psychiatric symptoms, and a history of substance use disorders. ${ }^{31,32}$ Another report suggested that cholesterol was linked to suicidal behavior due to its association with serotonergic activity, steroid regulation, and brain-derived neurotrophic factor levels. ${ }^{33}$

In this study, we found no cognitive performance differences between the two subgroups, which was inconsistent with our hypothesis. However, this result is consistent with a smaller previous study by our group (25 suicide attempters and 291 non-attempters), ${ }^{15}$ and another two studies on Caucasian chronic SCZ patients, ${ }^{34,35}$ which used other neurocognitive assessments than the RBANS. None of these studies found a difference in cognitive performance between SCZ patients with or without a history of suicide attempts. However, studies have found both negative and positive relationships between cognitive performance and suicide risk, ${ }^{8,36}$ which could be due to several reasons. Suicide attempters may have different neurocognitive characteristics than non-attempters, which suggests that comprehensive neurocognitive assessments are needed to investigate their relationship. ${ }^{37}$ Furthermore, the cognitive performance of SCZ patients varies over time. ${ }^{38}$ Meanwhile, impairments in the serotonin neurotransmitter system and the hypothalamicpituitary-adrenal axis stress response system significantly affect cognitive performance, ${ }^{39}$ which could lead to changes in cognitive function in SCZ patients, regardless of suicidal behavior. Thus, the relationship between suicide attempts and cognitive function must be further explored in future investigations.

There are several methodological limitations in the present study. First, we used a cross-sectional design, which prevented causal inferences between suicide attempts and the SCZ inpatient characteristics. Second, this study did not evaluate some important variables. Since data was not collected on the lethality and severity of suicide attempts, the association between patient characteristics and the severity of attempted suicide was not analyzed. Meanwhile, variables such as depressive symptoms, hopelessness, suicidal ideation, and the severity of suicide attempts were not evaluated by specific scales. Further investigations with reliable tools, such as the Hamilton Depression Scale and the Beck Scale for Suicide Ideation, will deepen our understanding. Third, since our participants were chronically hospitalized patients with longer illness duration and more severe psychopathology (including predominantly negative symptoms), who differ from typical psychiatric outpatients or patients in the community, our results cannot be extended to outpatients or community patients. Fourth, the gender imbalance (742 men and 166 women) was a limitation and could result in statistical bias. This limitation should be corrected in future investigations. Fifth, cultural factors are related to suicidal behavior and were not assessed in this study, which should be corrected in future studies. Finally, since the data on suicide attempts was acquired from interviews and medical records, recall bias cannot be completely excluded.

In summary, the findings of this study confirmed our previous research, i.e. a suicide attempt rate of approximately $10 \%$ among hospitalized Chinese Han SCZ patients, which is notably higher than the general population. The suicide rates found in this study and in other Chinese samples are similar; however, they are much lower than the estimated Western prevalence rate of $18-55 \%$. SCZ inpatients who had attempted suicide were, on average, younger, more likely to smoke, and had a longer illness duration, as well as more serious general psychological and depressive symptoms. However, we found no significant difference in cognitive performance between those who had and had not attempted suicide. However, due to the methodological limitations of this study, such as its cross-section design, lack of assessment for some important variables, its sample consisting mainly of chronically hospitalized patients from the Beijing area, its lack of cultural variables associated with suicide, and the possible recall basis, our findings should be considered preliminary and require further investigation. Longitudinal studies with large samples of patients are needed to elucidate the causal relationship between sociodemographic and clinical parameters, cognition, and suicide attempts in SCZ.

\section{Disclosure}

The authors report no conflicts of interest.

\section{References}

1 Ventriglio A, Gentile A, Bonfitto I, Stella E, Mari M, Steardo L, et al. Suicide in the early stage of schizophrenia. Front Psychiatry. 2016;7:116.

2 Hor K, Taylor M. Suicide and schizophrenia: a systematic review of rates and risk factors. J Psychopharmacol2010;24(4 Suppl)81-90.

3 Bolton C, Gooding P, Kapur N, Barrowclough C, Tarrier N. Developing psychological perspectives of suicidal behaviour and risk in people with a diagnosis of schizophrenia: we know they kill themselves but do we understand why?. Clin Psychol Rev. 2007;27: 511-36.

4 Hawton K, Sutton L, Haw C, Sinclair J, Deeks JJ. Schizophrenia and suicide: systematic review of risk factors. $\mathrm{Br} J$ Psychiatry. 2005; 187:9-20.

5 Barrett EA, Mork E, Faerden A, Nesvåg R, Agartz I, Andreassen OA, et al. The development of insight and its relationship with suicidality 
over one year follow-up in patients with first episode psychosis Schizophr Res. 2015;162:97-102.

6 Reutfors J, Brandt L, Jonsson EG, Ekbom A, Sparen P, Osby U. Risk factors for suicide in schizophrenia: findings from a Swedish population-based case-control study. Schizophr Res. 2009;108:231-7.

7 World Health Organization (WHO). Global health estimates 2016: deaths by cause, age, sex, by country and by region, 2000-2016 [Internet]. 2018 [cited 2020 Apr 8]. www.who.int/healthinfo/global burden_disease/estimates/en/.

8 Zhang $\overline{X Y}$, Du X, Yin G, Zhang Y, Chen D, Xiu M, et al. Prevalence and clinical correlates of and cognitive function at the time of suicide attempts in first-episode and drug-naive patients with schizophrenia. J Clin Psychiatry.2018 Jul 24;79(4)pii: 17m11797. doi: 10.4088/ JCP.17m11797.

9 Zhang XY, Al Jurdi RK, Zoghbi AW, Chen DC, Xiu MH, Tan YL, et al. Prevalence, demographic and clinical correlates of suicide attempts in Chinese medicated chronic inpatients with schizophrenia. J Psychiatr Res. 2013;47:1370-5.

10 Dong M, Wang SB, Wang F, Zhang L, Ungvari GS, $\mathrm{Ng} \mathrm{CH}$, et al. Suicide-related behaviours in schizophrenia in China: a comprehensive meta-analysis. Epidemiol Psychiatr Sci. 2019;28:290-9.

11 DeJong TM, Overholser JC, Stockmeier CA. Apples to oranges? A direct comparison between suicide attempters and suicide completers. J Affect Disord. 2010;124:90-7.

12 Huber RS, Hodgson R, Yurgelun-Todd DA. A qualitative systematic review of suicide behavior using the cognitive systems domain of the research domain criteria (RDoC) framework. Psychiatry Res. 2019; 282:112589.

13 Togay B, Noyan H, Tasdelen R, Ucok A. Clinical variables associated with suicide attempts in schizophrenia before and after the first episode. Psychiatry Res. 2015;229:252-6.

14 Adan A, Capella MD, Prat G, Forero DA, López-Vera S, Navarro JF. Executive functioning in men with schizophrenia and substance use disorders. Influence of lifetime suicide attempts. PloS One. 2017;12 e0169943.

15 Zoghbi AW, Al Jurdi RK, Deshmukh PR, Chen DC, Xiu MH, Tan YL, et al. Cognitive function and suicide risk in Han Chinese inpatients with schizophrenia. Psychiatry Res. 2014;220:188-92.

16 Kao YC, Liu YP, Cheng TH, Chou MK. Cigarette smoking in outpatients with chronic schizophrenia in Taiwan: relationships to sociodemographic and clinical characteristics. Psychiatry Res. 2011; 190:193-9.

17 Kay SR, Fiszbein A, Opler LA. The positive and negative syndrome scale (PANSS) for schizophrenia. Schizophr Bull. 1987:13:261-76.

18 Wallwork RS, Fortgang R, Hashimoto R, Weinberger DR, Dickinson D. Searching for a consensus five-factor model of the positive and negative syndrome scale for schizophrenia. Schizophr Res. 2012; 137:246-50.

19 Zhang BH, Tan YL, Zhang WF, Wang ZR, Yang GG, Shi C, et al. Repeatable battery for the assessment of neuropsychological status as a screening test in Chinese: reliability and validity. Chin Ment Health J. 2008;22:865-9.

20 Lee S, Fung SC, Tsang A, Liu ZR, Huang YQ, He YL, et al. Lifetime prevalence of suicide ideation, plan, and attempt in metropolitan China. Acta Psychiatr Scand. 2007;116:429-37.

21 Law S, Liu P. Suicide in China: unique demographic patterns and relationship to depressive disorder. Curr Psychiatry Rep. 2008;10:80-6.

22 Phillips MR, Yang G, Li S, Li Y. Suicide and the unique prevalence pattern of schizophrenia in mainland China:a retrospective observational study. Lancet. 2004;364:1062-8.
23 Barrett EA, Sundet K, Simonsen C, Agartz I, Lorentzen S, Mehlum L, et al. Neurocognitive functioning and suicidality in schizophrenia spectrum disorders. Compr Psychiatry. 2011;52:156-63.

24 Fuller-Thomson E, Hollister B. Schizophrenia and suicide attempts: findings from a representative community-based Canadian sample. Schizophr Res Treatment. 2016;2016:3165243.

25 Yan F, Xiang YT, Hou YZ, Ungvari GS, Dixon LB, Chan SS, et al. Suicide attempt and suicidal ideation and their associations with demographic and clinical correlates and quality of life in Chinese schizophrenia patients. Soc Psychiatry Psychiatr Epidemiol. 2013;48:447-54.

26 Li Q, Xiang YT, Su YA, Shu L, Yu X, Correll CU, et al. Clozapine in schizophrenia and its association with treatment satisfaction and quality of life: findings of the three national surveys on use of psychotropic medications in China (2002-2012). Schizophr Res. 2015;168:523-9.

27 Zalsman G, Hawton K, Wasserman D, van Heeringen K, Arensman $E$, Sarchiapone $M$, et al. Suicide prevention strategies revisited: 10-year systematic review. Lancet Psychiatry. 2016;3:646-59.

28 Tovilla-Zarate C, Juarez-Rojop I, Ramon-Frias T, Villar-Soto M, PoolGarcía S, Medellín BC, et al. No association between COMT val158met polymorphism and suicidal behavior: meta-analysis and new data. BMC Psychiatry. 2011;11:151.

29 Sokolowski M, Wasserman J, Wasserman D. Polygenic associations of neurodevelopmental genes in suicide attempt. Mol Psychiatry. 2016;21:1381-90.

30 Evins AE, Korhonen T, Kinnunen TH, Kaprio J. Prospective association between tobacco smoking and death by suicide: a competing risks hazard analysis in a large twin cohort with 35-year follow-up. Psychol Med. 2017;47:2143-54.

31 Fleischhacker WW, Kane JM, Geier J, Karayal O, Kolluri S, Eng SM, et al. Completed and attempted suicides among 18,154 subjects with schizophrenia included in a large simple trial. J Clin Psychiatry. 2014;75:e184-90.

32 Ostergaard ML, Nordentoft M, Hjorthoj C. Associations between substance use disorders and suicide or suicide attempts in people with mental illness: a Danish nation-wide, prospective, register-based study of patients diagnosed with schizophrenia, bipolar disorder, unipolar depression or personality disorder. Addiction. 2017;112:1250-9.

33 Cantarelli Mda G, Tramontina AC, Leite MC, Goncalves CA. Potential neurochemical links between cholesterol and suicidal behavior. Psychiatry Res. 2014;220:745-51.

$34 \mathrm{Kim} \mathrm{CH}$, Jayathilake K, Meltzer HY. Hopelessness, neurocognitive function, and insight in schizophrenia: relationship to suicidal behavior. Schizophr Res. 2003;60:71-80.

35 Potkin SG, Anand R, Alphs L, Fleming K. Neurocognitive performance does not correlate with suicidality in schizophrenic and schizoaffective patients at risk for suicide. Schizophr Res. 2003;59:59-66.

36 Verma D, Srivastava MK, Singh SK, Bhatia T, Deshpande SN. Lifetime suicide intent, executive function and insight in schizophrenia and schizoaffective disorders. Schizophr Res. 2016;178:12-6.

37 Canal-Rivero M, Lopez-Morinigo JD, Setien-Suero E, Ruiz-Veguilla M, Ayuso-Mateos JL, Ayesa-Arriola R, et al. Predicting suicidal behaviour after first episode of non-affective psychosis: the role of neurocognitive functioning. Eur Psychiatry. 2018;53:52-7.

38 Dickerson F, Schroeder J, Stallings C, Origoni A, Katsafanas E, Schwienfurth LA, et al. A longitudinal study of cognitive functioning in schizophrenia: clinical and biological predictors. Schizophr Res. 2014;156:248-53.

39 van Heeringen K, Mann JJ. The neurobiology of suicide. Lancet Psychiatry. 2014;1:63-72. 